\title{
Back to basics: food for thought—and nutrition
}

\author{
Stephen B Hanauer
}

In his article "Unhappy Meals" in The New York Times Magazine of 28 January 2007, Michael Pollan (Knight Professor of Journalism at the University of California, Berkeley, and author of the book The Omnivore's Dilemma) begins by stating "Eat food. Not too much. Mostly plants." This is his answer to the question of "...what we humans should eat in order to be maximally healthy." In this essay, he describes how the food industry, nutritional science and journalism have, together, complicated the question of what we should eat, and how governments - which are often influenced by these three groups-have contributed to the confusion.

Pollan describes how society has narrowed its focus from foods to nutrients and, in doing so, can no longer see the forest for the trees. From our initial recognition of the macronutrients (protein, fat and carbohydrates) in food to the identification of specific micronutrient deficiencies (which led to the identification of vitamins), we have moved into an age of 'nutritionism', in which we concentrate on eating nutrients rather than foods.

I was overwhelmed to read how nutrition in the US became politicized. In 1977, the US Senate Select Committee on Nutrition and Health published a document entitled Dietary Goals for the United States (also known as the McGovern report), after holding hearings on the rising incidence of heart disease, cancer and diabetes. As epidemiologic data indicated that meat and dairy consumption contributed to heart disease, the Committee's draft recommendations included advice to reduce dietary intakes of red meat and dairy products. Pressure from industry lobbyists led to the statement that Americans should "reduce consumption of meat" being rewritten as "Choose meats, poultry and fish that will reduce saturated fat intake." This change led to a focus on saturated fat and subsequently on other nutrients, rather than whole foods, and the development of nutritionism founded on ideology, not science. ...society has

narrowed its

focus from

foods to

nutrients and,

in doing so,

can no longer

see the forest

for the trees

SB Hanauer is Editorin-Chief of Nature Clinical Practice Gastroenterology \& Hepatology.

\section{Competing interests}

The author declared he has no competing interests.

www.nature.com/clinicalpractice doi:10.1038/ncpgasthep0784
This shift in focus from foods to nutrients has led to the development of multibillion-dollar industries, including those involved with health supplements, vitamins and processed foods. The processed-food industry markets inexpensive calories, with the inclusion or exclusion of specific macronutrients or micronutrients. Instead of improving the health of Americans, however, this shift in focus has coincided with an increasing incidence of obesity, diabetes, heart disease and some cancers.

As Pollan describes, diverse cultures have evolved to eat different diets that are suited to local food supplies and growing conditions. Despite this diversity, most societies have evolved towards a healthy dietary equilibrium, as long as food availability is maintained. Yet, when cultures diverge from their historical diet because of changes in growing conditions, shortages, or adoption of nontraditional foods (e.g. the 'Western' diet) negative health consequences ensue. The worldwide simplification of the food chain has resulted in a significant decline in the nutritional quality of produce, and excessive food processing has led to an increase in the consumption of seeds and grains at the expense of leaves. These shifts have led to overconsumption of macronutrients and, conversely, to a reduction in the consumption of critical micronutrients that necessitates supplementation. Despite the addition of dietary supplements, however, there are little data on the absorption and interactions of individual nutrients (e.g. vitamins, minerals, antioxidants) that would normally be delivered by whole foods. Indeed, the health benefits ascribed to vitamins and other supplements administered separately from a balanced diet of real foods have not been substantiated by epidemiological studies.

Pollan quotes Hippocrates' injunction to "...let food be thy medicine." It seems that we have come full circle with dietary advice, and it is time to get back to basics. 